\title{
Estudo Comparativo de Modelos Computacionais Gerados sobre Representações de Imagens de Coloscopia: Tecido de Mucosa Normal VS Tecido de Mucosa de Pólipo Cólico
}

\author{
Comparative Study of Computacional Models Generated from \\ Representations of Colonoscopic Images: Normal Mucosal Tissues VS \\ Mucosal Tissues of Colic Polyp
}

\begin{abstract}
CARLOS ANDRES FERRERO ${ }^{1}$, HUEI DIANALEE ${ }^{2}$, NEWTON SPOLAÔR ${ }^{3}$, CLÁUDIO SADDY RODRIGUES COY ${ }^{4}$, JOÃO JOSÉ FAGUNDES ${ }^{5}$, RENATO BOBSIN MACHADO ${ }^{6}$, EVERTON ALVARES CHERMAN ${ }^{3}$, FENG CHUNG WU ${ }^{7}$
\end{abstract}

\begin{abstract}
${ }^{1}$ Pesquisador do Laboratório de Bioinformática (LABI), Universidade Estadual do Oeste do Paraná (UNIOESTE), Parque Tecnológico Itaipu (PTI), Foz do Iguaçu, PR, Brasil; ${ }^{2}$ Professora Doutora e Coordenadora do Curso de Ciência da Computação da UNIOESTE e Coordenadora Geral do LABI - UNIOESTE, Foz do Iguaçu, PR, Brasil; ${ }^{3}$ Estagiário do Laboratório de Bioinformática (LABI), Universidade Estadual do Oeste do Paraná (UNIOESTE), Parque Tecnológico Itaipu (PTI), Foz do Iguaçu, PR, Brasil; ${ }^{4}$ Professor Doutor do Departamento de Cirurgia (D.M.A.D.), Serviço de Coloproctologia da Universidade Estadual de Campinas (UNICAMP), Campinas, SP, Brasil; ${ }^{5}$ Professor Doutor do Departamento de Cirurgia da Faculdade de Ciências Médicas da UNICAMP, Campinas, SP, Brasil; ${ }^{6}$ Professor e Coordenador da Área Computacional do LABI - UNIOESTE, Foz do Iguaçu, PR, Brasil; ${ }^{7}$ Pesquisador do Serviço de Coloproctologia da Faculdade de Ciências Médicas da UNICAMP, Campinas, SP, Brasil. Professor Doutor da UNIOESTE e Coordenador da Área Médica do LABI - UNIOESTE, Foz do Iguaçu, PR, Brasil.
\end{abstract}

FERRERO CA; LEE HD; SPOLAÔR N; COY CSR; FAGUNDES JJ; MACHADO RB; CHERMAN EA; WU FC. Estudo Comparativo de Modelos Computacionais Gerados sobre Representações de Imagens de Coloscopia: Tecido de Mucosa Normal VS Tecido de Mucosa de Pólipo Cólico. Rev bras Coloproct, 2009;29(1): 023-029.

RESUMO: Objetivo: analisar a qualidade preditiva de modelos computacionais para a diferenciação de tecidos cólicos, construídos a partir da representação de Imagens de Coloscopia (IC) como Matrizes de Co-ocorrência (MC). Materiais e Métodos: os modelos foram construídos aplicando técnicas de análise de imagens e de inteligência artificial. Foram utilizadas 67 IC, contendo pólipos, a partir das quais foram extraídas uma imagem da parte de tecido de pólipo e outra de tecido sem pólipo adjacente, totalizando 134 imagens. Para cada imagem, foram construídas MC para diferentes valores do parâmetro distância, $\mathrm{D}=1$ a 5, e extraídas 11 características de textura. Com essa representação, foram criados cinco modelos computacionais baseados em árvores de decisão. Os modelos foram avaliados utilizando: (a) validação cruzada e (b) tabelas de contingência. Resultados: na análise (a), o modelo de $D=3$ apresentou o menor erro médio (22,25\% $\pm 11,85 \%)$. Na análise (b), os modelos de $D=1$ e 3 apresentaram os melhores valores de precisão. Conclusão: os valores do parâmetro de distância $D=1$ e 3 apresentaram os modelos com as melhores qualidades preditivas. Os resultados mostraram que os modelos construídos apresentaram-se promissores para a construção de sistemas computacionais de suporte à decisão.

Descritores: Colonoscopia, Polipose Intestinal, Neoplasias do Cólon, Inteligência Artificial, Interpretação de Imagem Assistida por Computador.

Trabalho realizado no Laboratório de Bioinformática (LABI), Universidade Estadual do Oeste do Paraná (UNIOESTE), Parque Tecnológico Itaipu (PTI), e no Serviço de Coloproctologia (SC) da Faculdade de Ciências Médicas (FCM) da Universidade Estadual de Campinas (UNICAMP).

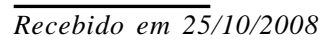

Aceito para publicação em 13/11/2008

Conflito de Interesses: nenhum

Fonte de auxílio à pesquisa: Programa de Desenvolvimento Tecnológico Avançado - Fundação Parque Tecnológico Itaipu (PDTA/FPTI-BR). Termos de Concessão de Bolsa 009/2007, 041/2008 e 087/2008. Fundação de Apoio ao Desenvolvimento Científico e Tecnológico do Paraná (ARAUCARIA). Convênio 051/2007. 

Carlos Andres Ferrero e Cols.

\section{INTRODUÇÃO}

Exames médicos são registrados, frequentemente, em hospitais e clínicas médicas, por meio da utilização de Sistemas de Gerenciamento de Dados, os quais possibilitam que registros com informações de pacientes e de exames relacionados, sejam armazenados e consultados pelos especialistas. A descrição desses exames pode estar representada em diversos formatos, como texto, imagem e vídeo ${ }^{(1,2)}$, com o objetivo de complementar os laudos médicos realizados pelos especialistas e, desse modo, prover uma descrição mais completa a respeito do estado de saúde do paciente.

Nesse sentido, imagens médicas provenientes de exames coloscópicos, por exemplo, têm sido comumente armazenadas com o intuito de prover esse complemento e dar suporte aos especialistas no diagnóstico de enfermidades. Ainda, essas imagens, podem ser analisadas por processos computacionais que permitam extrair informações relevantes a respeito das anormalidades evidenciadas por meio desse exame. Duas áreas para prover suporte a essa tarefa são: (1) Análise de Imagens e (2) Inteligência Computacional. A primeira refere-se à extração de informações relevantes em imagens e, a segunda, à busca de padrões e à construção de modelos ${ }^{(3)}$, os quais permitem classificar imagens nunca antes classificadas.

No Brasil, segundo o Instituto Nacional do Câncer (INCA), o câncer colorretal constitui uma das neoplasias malignas de maior incidência na população, ocupando o quarto lugar em relação ao sexo masculino e o terceiro lugar em relação ao sexo feminino ${ }^{(4)}$. De acordo com o INCA, no ano de 2008, haverá aproximadamente 12.490 casos desse tipo de câncer em homens e 14.500 em mulheres. Em relação à mortalidade média mundial, o câncer de cólon e reto, não apresenta grandes diferenças entre países desenvolvidos e países em desenvolvimento ${ }^{(4)}$. A coloscopia apresentase como uma ferramenta indispensável para o diagnóstico de doenças do intestino grosso, o que torna importante a análise computacional para a identificação automática de anormalidades evidenciadas por esse exame médico.

O presente trabalho está inserido em um projeto maior, de Análise de Imagens Médicas, o qual é desenvolvido em uma parceria entre o LABI/ UNIOESTE e o SC/FCM/UNICAMP. Nesse contexto, este trabalho tem como objetivo analisar a qualidade preditiva de modelos computacionais para a dife- renciação de tecidos cólicos, construídos a partir da representação de Imagens de Coloscopia (IC) como Matrizes de Co-ocorrência (MC).

\section{MATERIAIS E MÉTODOS}

Para a realização deste trabalho, 67 imagens de exames de coloscopia, provenientes do SC/FCM/ UNICAMP, referentes a pólipos do tipo Protruso Pediculado (de acordo com os padrões da Sociedade Japonesa do Câncer Colorretal), foram submetidas a uma metodologia que possibilita a extração de características intrínsecas de fragmentos de imagem de tecido cólico e a busca de padrões para a construção de modelos de classificação ${ }^{(5)}$. Essa metodologia, a qual é apoiada por um sistema computacional desenvolvido no LABI/UNIOESTE, denominado Medical Image Analysis System (MIAS) ${ }^{(5,6)}$, é constituída de quatro etapas: (1) coleta do Conjunto de Imagens (CI); (2) construção do Vetor de Características (VC); (3) construção de modelos de classificação; e (4) avaliação de modelos.

Na Etapa (1) foi realizada a coleta do conjunto de imagens para análise. Para cada imagem foram selecionadas uma parte de imagem de mucosa normal e outra de pólipo cólico (Figura 1). Desse modo, foi construído o CI contendo 134 imagens, cada uma referente a uma das partes selecionadas. Na Etapa (2) essas imagens foram submetidas ao processo de extração de características. Neste trabalho foram extraídas características de textura, baseadas em MC, as quais representam as transições de nível de intensidade entre pontos da imagem considerando uma distância e uma direção ${ }^{(2)}$.

$\mathrm{Na}$ figura 2, é apresentado um exemplo de construção de $\mathrm{MC}$ a partir de uma imagem de 5 x 5 pontos e 3 níveis de intensidade ${ }^{(2)}$. Na MC, as Linhas (L) e Colunas (C) representam os níveis de intensidade, e cada posição da matriz corresponde ao número de transições do nível de cinza L para nível de cinza $C$ considerando direção $0^{\circ}$ e distância $\mathrm{D}=1$. Desse modo, foram extraídas 11 características de textura cujos valores foram calculados a partir das MC. Essas características são representadas pelo: segundo momento angular, pelo contraste, pela correlação, por meio da variância, da entropia, do momento inverso da diferença, da soma da média, da soma da variância, da soma da entropia, da diferença da variância e da diferença da entropia ${ }^{(7)}$. A extração dessas características requer 

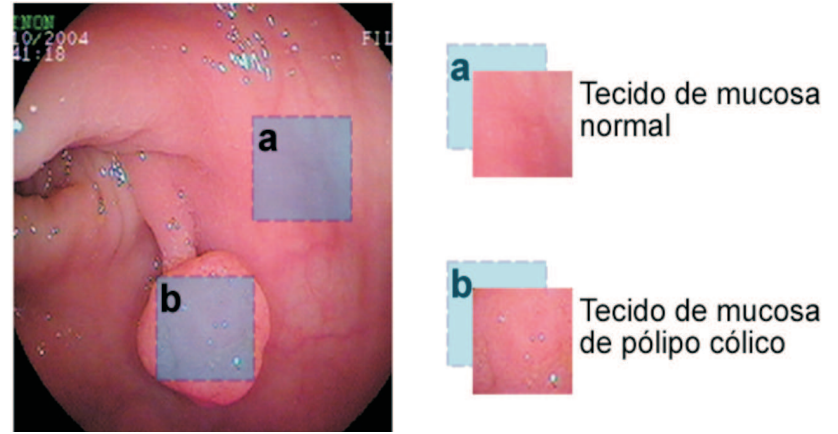

Figura 1 - Seleção de fragmentos de tecido de mucosa normal e de pólipo cólico.

\section{Imagem}

\begin{tabular}{|l|l|l|l|l|}
\hline $\mathbf{0}$ & $\mathbf{0}$ & $\mathbf{0}$ & $\mathbf{1}$ & $\mathbf{2}$ \\
\hline $\mathbf{1}$ & $\mathbf{1}$ & $\mathbf{0}$ & $\mathbf{1}$ & $\mathbf{1}$ \\
\hline 2 & 2 & 1 & 0 & $\mathbf{0}$ \\
\hline $\mathbf{1}$ & $\mathbf{1}$ & $\mathbf{0}$ & $\mathbf{2}$ & $\mathbf{0}$ \\
\hline $\mathbf{1}$ & $\mathbf{0}$ & $\mathbf{1}$ & $\mathbf{0}$ & $\mathbf{0}$ \\
\hline
\end{tabular}

Figura 2 - Imagem representada em três níveis de cinza como uma matriz de pixels $5 \times 5$ e a respectiva $M C$ para a direção $0^{\circ}$ e a distância 1 .

a definição de três parâmetros: (a) número de níveis de cinza da imagem ou qualidade, representada por $\mathrm{L}$ ou C; (b) a distância D a ser considerada, que identifica o grau de aproximação da imagem; e (c) a direção, que indica a angulação de análise da imagem.

Ainda na Etapa (2), a partir das partes contidas em CI foram construídas cinco MC para cada parte, considerando valores de distância $\mathrm{D}=1,2,3,4$ e 5, e extraídas as 11 características de textura mencionadas, para representar cada uma das partes a partir de um VC. Em relação à qualidade da imagem, foi determinada a não variação deste parâmetro, o qual foi fixado em 64 níveis de cinza. Assim, foram construídos cinco conjuntos de dados, $\mathrm{CD}_{1}, \mathrm{CD}_{2}, \mathrm{CD}_{3}, \mathrm{CD}_{4}, \mathrm{CD}_{5}$, os quais representam cada valor do parâmetro de distância considerado. Desse modo, os valores dos atributos de cada exemplo, desses conjuntos de dados, correspondem à média dos valores das características extraídas para as direções $0,45,90$ e 135 graus.

Na Etapa (3) foram construídos modelos aplicando métodos de Aprendizado de Máquina, uma subárea da Inteligência Computacional que possibilita a busca de padrões em grandes conjuntos de dados e permite a geração de modelos de classificação. Um dos algoritmos computacionais para a geração de modelos é o J48, o qual consiste em uma implementação do algoritmo $\mathrm{C} 4.5^{(8)}$, disponibilizado pela ferramenta computacional WEKA ${ }^{1}$. Esse algoritmo consiste na divisão de conjuntos de dados multidimensionais a partir do traçado de sucessivos hiperplanos, que permitam diferenciar as classes envolvidas no problema. Desse modo, nessa etapa os modelos são construídos com o objetivo de diferenciar partes de tecido cólico normal de partes de tecido de pólipo cólico.

Ao final, na Etapa (4), foi realizada a avaliação dos modelos construídos na etapa anterior. Primeiramente foi utilizada a técnica de validação cruzada com dez partições ${ }^{(9)}$, para obter uma aproximação do erro verdadeiro do modelo quanto à capacidade de predição. Posteriormente, foram construídas as tabelas de contingência para cada modelo, no intuito de extrair as medidas de Valor Preditivo Positivo (VPP), Valor Preditivo Negativo (VPN), Sensibilidade (S) e Especificidade $(\mathrm{E})^{(9)}$. A análise estatística foi realizada por meio do aplicativo GraphPad InStat ${ }^{\circledR 2}$, aplicandose o teste $t$ student considerando um nível de significância de $95 \%$ ( $p$-valor $=0,05$ ).

\section{RESULTADOS}

A tabela 1 apresenta a avaliação da precisão preditiva dos modelos construídos considerando $\mathrm{D}=1$, 2, 3, 4 e 5, de acordo com a análise dos valores de erro médio e de desvio-padrão obtidos pela validação cruzada. Essa tabela também apresenta os $p$-valores resultantes do teste estatístico para verificar a seleção do modelo de maior precisão.

Nas tabelas 2, 3, 4, 5 e 6, são apresentadas as tabelas de contingência dos modelos construídos considerando $\mathrm{D}=1,2,3$, 4 e 5, respectivamente.

Os valores de VPP, VPN, S e E correspondentes a cada modelo computacional construído são apresentados na tabela 7 .

\section{DISCUSSÃO}

$\mathrm{Na}$ análise de imagens médicas é frequente a descrição de imagens conforme propriedades baseadas em cor, textura e forma ${ }^{(10)}$. Em trabalho anterior analisado $^{(11)}$ foi realizada a classificação de exames de coloscopia de pacientes com câncer colorretal e pacientes sem essa enfermidade. As imagens coletadas foram representadas por meio de características glo- 
Tabela 1 - Avaliação preditiva dos modelos construídos considerando as distâncias $D=1,2,3,4$ e 5 .

\begin{tabular}{lrrrrr}
\hline & \multicolumn{5}{c}{ Distância } \\
\cline { 2 - 6 } & $\mathbf{1}$ & $\mathbf{2}$ & $\mathbf{3}$ & $\mathbf{4}$ & $\mathbf{5}$ \\
\hline Precisão média & 23,901 & 28,297 & 22,253 & 29,011 & 30,604 \\
Desvio-padrão & 12,366 & 12,216 & 11,850 & 10,084 & 8,369 \\
$p$-valor $t$ student & 0,550 & 0,131 & - & 0,048 & 0,101 \\
\hline
\end{tabular}

Tabela 2 - Tabela de contingência do modelo construído considerando distância 1.

\begin{tabular}{lccc}
\hline & \multicolumn{2}{c}{ Classificação pelo modelo } & \\
Tecido & Tecido de pólipo & Tecido & sem pólipo \\
\hline De pólipo & 46 & 21 & Total \\
Sem pólipo & 11 & 56 & 67 \\
Total & 57 & 77 & 67 \\
\hline
\end{tabular}

Tabela 3 - Tabela de contingência do modelo construído considerando distância 2.

\begin{tabular}{lccc}
\hline & \multicolumn{2}{c}{ Classificação pelo modelo } & \\
Tecido & Tecido de pólipo & Tecido sem pólipo & Total \\
\hline De pólipo & 47 & 20 & 67 \\
Sem pólipo & 18 & 49 & 67 \\
Total & 65 & 69 & 134 \\
\hline
\end{tabular}

bais extraídas a partir de MC. No entanto, para a identificação de tecidos de pólipos em imagens de coloscopia é essencial a realização de uma análise local, pois por meio desse procedimento, tecidos cólicos normais e anormais poderão ser diferenciados em uma mesma imagem. Sob esse escopo, foi realizada, em trabalho anterior ${ }^{(12)}$, a análise local de imagens de coloscopia contendo pólipos, utilizando o espectro de textura da imagem. Assim, essas imagens foram classificadas de acordo com a similaridade identificada entre os espectros de textura de tecidos de pólipo e de tecidos com ausência de pólipo. Todavia, confrontando as características de análise do trabalho anterior com o atualmente citado $^{(6)}$, neste último, as imagens foram submetidas ao processo de extração de características a partir das MC e avaliado o parâmetro de qualidade da imagem, isto é, o número de níveis de cinza a ser considerado na análise (parâmetro (a)). Com isso, concluiu-se que a qualidade da imagem e o tempo computacional de processamento são diretamente pro-

Tabela 4 - Tabela de contingência do modelo construído considerando distância 3.

\begin{tabular}{lccc}
\hline & \multicolumn{2}{c}{ Classificação pelo modelo } & \\
Tecido & Tecido de pólipo & Tecido sem pólipo & Total \\
\hline De pólipo & 53 & 14 & 67 \\
Sem pólipo & 16 & 51 & 67 \\
Total & 69 & 65 & 134 \\
\hline
\end{tabular}


Tabela 5 - Tabela de contingência do modelo construído considerando distância 4.

\begin{tabular}{lccc}
\hline & \multicolumn{2}{c}{ Classificação pelo modelo } & \\
Tecido & Tecido de pólipo & Tecido & sem \\
pólipo & Total \\
\hline De pólipo & 50 & 17 & 67 \\
Sem pólipo & 22 & 45 & 67 \\
Total & 72 & 62 & 134 \\
\hline
\end{tabular}

Tabela 6 - Tabela de contingência do modelo construído considerando distância 5.

\begin{tabular}{|c|c|c|c|}
\hline \multicolumn{4}{|c|}{ Classificação pelo modelo } \\
\hline Tecido & Tecido de pólipo & Tecido sem pólipo & Total \\
\hline De pólipo & 51 & 16 & 67 \\
\hline Sem pólipo & 25 & 42 & 67 \\
\hline Total & 76 & 58 & 134 \\
\hline
\end{tabular}

Tabela 7 - Valores calculados a partir da tabela de contingência.

\begin{tabular}{ccccc}
\hline Distância & VPP & VPN & S & E \\
\hline 1 & $80,70 \%$ & $72,73 \%$ & $68,66 \%$ & $83,58 \%$ \\
2 & $72,31 \%$ & $71,01 \%$ & $70,15 \%$ & $73,13 \%$ \\
3 & $76,81 \%$ & $78,46 \%$ & $79,10 \%$ & $76,12 \%$ \\
4 & $69,44 \%$ & $72,58 \%$ & $74,63 \%$ & $67,16 \%$ \\
5 & $67,11 \%$ & $72,41 \%$ & $76,12 \%$ & $62,69 \%$ \\
\hline
\end{tabular}

porcionais. Entretanto, é importante ressaltar que a qualidade de imagem não representa diretamente a precisão do modelo de classificação, pois diferentes valores para cada característica podem influenciar na ordem em que o algoritmo J48 escolhe os atributos para a criação dos hiperplanos, o que resulta na construção de árvores de decisão com estruturas diferentes. De acordo com essas considerações o parâmetro (a), de qualidade da imagem, foi fixado em 64 níveis de cinza. Para avaliar a capacidade do método para construir modelos que permitam classificar tecidos de mucosa cólica de pólipo e sem pólipo, o parâmetro (b), de distância, foi avaliado neste trabalho.

A análise dos resultados, considerando a precisão preditiva obtida pelo método de validação cruza$\mathrm{da}$, indicou que o modelo construído com $\mathrm{D}=3$ apresentou o menor erro médio, enquanto que o modelo correspondente ao valor de $\mathrm{D}=5$ apresentou o menor desvio-padrão. Ainda, o valor desse desvio padrão di- minuiu quando a distância aproximou-se de $\mathrm{D}=5$. A partir destes resultados, constatou-se a necessidade de verificar a existência de diferença estatisticamente significativa entre os modelos, de modo que se possa comprovar, ou refutar, a superioridade do modelo que considera $\mathrm{D}=3$ em relação à precisão preditiva de cada um dos outros modelos construídos. Os erros médios considerados foram submetidos e aceitos no teste de normalidade, o que permitiu a aplicação do teste estatístico $t$. Este teste foi realizado com nível de significância de $95 \%$ para amostras emparelhadas.

Os $p$-valores obtidos a partir da aplicação do teste $t$ para os modelos construídos com $\mathrm{D}=1,2$, 4 e 5 foram de $0,550,0,131,0,048$ e 0,101 , respectivamente, conforme é apresentado na Tabela 1. Desse modo, o teste permitiu evidenciar diferença estatisticamente significativa apenas entre o modelo $\mathrm{D}=3$ e $\mathrm{D}=$ 4 , sendo que, em relação aos outros modelos construídos, os $p$-valores foram maiores do que 0,05 . 
Estudo Comparativo de Modelos Computacionais Gerados sobre Representações de Imagens de Coloscopia: Tecido de Mucosa Normal VS Tecido de Mucosa de Pólipo Cólico Carlos Andres Ferrero e Cols.
Com relação aos valores calculados a partir das tabelas de contingência (Tabela 7), o modelo construído com $\mathrm{D}=1$ apresentou as medidas VPP e E maiores que os outros modelos. Desse modo, foi evidenciada a capacidade do modelo de classificar fragmentos de tecidos de pólipo cólico como sendo dessa classe e de que, dado um fragmento de tecido de mucosa cólica normal, este seja classificado como tal. Por outro lado, em relação às medidas VPN e S, o modelo considerando $\mathrm{D}=3$ apresentou os maiores valores de probabilidade. Esse fato evidenciou a maior probabilidade de acerto na classificação de tecidos de mucosa cólica normal e de que, dado um fragmento de tecido de mucosa de pólipo, este seja classificado como tal.

A diferença entre a qualidade dos modelos em relação às medidas das tabelas de contingência pode ser verificada mais especificamente pelas tabelas $2 \mathrm{e}$ 4 , referentes às tabelas de contingência dos modelos gerados com $\mathrm{D}=1$ e 3 , respectivamente, para fragmentos de tecido de mucosa de pólipo cólico e normal. Esses valores indicam que, de um total de 67 fragmentos de cada classe, o modelo de $\mathrm{D}=1$ identificou 46 fragmentos de mucosa de pólipo cólico e 56 normais, enquanto o modelo de $\mathrm{D}=3$ identificou 53 e 51, respectivamente.

Com relação à precisão de classificação dos modelos, considerando $\mathrm{D}=1$, dos 57 fragmentos classificados como de pólipo cólico, 46 foram corretos e dos 77 classificados como sem pólipo cólico, 56 foram corretos. Em relação à distância $\mathrm{D}=3$, dos 69 classificados como de pólipo cólico, 53 foram classificados corretamente e dos 65 classificados sem pólipo cólico, 51 foram classificados corretamente.

É importante ressaltar que não está sendo utilizada a forma estrutural do pólipo na imagem para análise, sendo apenas considerada a textura interna (Figura 1) dessa lesão tecidual. Desse modo, por meio da utilização de técnicas computacionais, foi possível representar esta estrutura por um conjunto de características que descrevem a textura do pólipo na imagem.

\section{CONCLUSÃO}

De acordo com os modelos construídos para a avaliação do parâmetro de distância, foi possível evidenciar a presença de padrões pictóricos de textura nos fragmentos de tecido pertencente à mucosa do pólipo cólico e à mucosa normal constituinte da parede do cólon. Desse modo, o valor de distância $\mathrm{D}=3$ apresentou-se como o mais adequado em relação à precisão preditiva, embora seja possível provar uma diferença estatisticamente significativa desse modelo apenas em relação ao modelo de $\mathrm{D}=4$. Além disso, esse modelo apresentou-se mais preciso em relação às medidas de VPN e $\mathrm{S}$, enquanto o modelo de $\mathrm{D}=1$ apresentou-se o mais adequado para as medidas de VPP e E. É relevante salientar que o modelo referente à $\mathrm{D}=$ 3 apresentou o segundo melhor valor de precisão para as duas medidas. Com isso, a representação de fragmentos de tecidos cólicos por meio de atributos de textura poderá ser promissora para o auxílio na construção de modelos computacionais que permitam a diferenciação entre tecidos de mucosa de pólipo e normal.

\section{AGRADECIMENTOS}

Ao Programa de Desenvolvimento Tecnológico Avançado (PDTA/FPTI-BR) e à Fundação de Apoio ao Desenvolvimento Científico e Tecnológico do Paraná (ARAUCARIA).

\footnotetext{
ABSTRACT: Purpose: to evaluate the predictive quality of computational models to differentiate colic tissues, based on Cooccorrurence Matrices (MC) representation of Coloscopic Images (IC). Materials and Methods: image analysis and artificial intelligence methods were employed to construct computational models. Sixty seven IC images, containing polyp, were considered in this work, from which a part containing a polypus and another without it were collected given origin to 134 images. For each one of these, different MC were constructed considering five distance parameters $(D=1$ to 5$)$ and the extraction of 11 texture characteristics. With this representation, five computational models were generated based on decision trees. These models were evaluated using two techniques: (a) cross-validation and (b) contingency tables. Results: for the (a) analysis, the model with $D=3$ presented the smaller average error $(22.25 \% \pm 11.85 \%)$. For the $(b)$ analysis, models with $D=$ 1 and 3 presented the best precision values. Conclusion: parameters $D=1$ and 3 presented models with the best predictive qualities. Results showed that the constructed models were promising to be applied within decision making computational systems.
}

Key words: Colonoscopy, Intestinal Polyposis, Colonic Neoplasms, Artificial Intelligence, Image Interpretation. 


\section{REFERÊNCIAS}

1. Karkanis S, Galoussi K, Maroulis D. Classification of Endoscopic Images Based on Texture Spectrum. Advance Course in Artificial Intelligence. Proceedings of Workshop on Machine Learning in Medical Applications; 1999; Chania, Grecia; p. 63-7.

2. Felipe JC, Traina AJM, Traina C. Retrieval by Content of Medical Images Using Texture for Tissue Identification. Proceeding of the 16th IEEE Symposium on Computer-based Medical Systems; 2003; New York, USA; p 175-6.

3. Rezende SO. Sistemas Inteligentes: Fundamentos e Aplicações. Barueri (SP), Brasil: Editora Manole; 2003.

4. Instituto Nacional de Câncer (INCA). Estimativa 2008: Incidência de Câncer no Brasil. Rio de Janeiro (RJ), Brasil; 2007.

5. Ferrero CA, Lee HD, Cherman E A, Coy CSR, Fagundes JJ, Góes JRN, et al. Utilização de Atributos Baseados em Textura para a Caracterização de Tecidos Cólicos em Imagens de Colonoscopia. Anais do VII Workshop de Informática Médica. 2007; Porto de Galinhas (PE), Brasil; p. 204-213.

6. Ferrero CA, Spolaôr N, Lee HD, Coy CSR, Fagundes JJ, Wu FC. Estudo Comparativo de Matrizes de Co-ocorrência em Análise de Imagens Médicas: Diferenciação de Tecidos Cólicos. Anales del $11^{\circ}$ Simposio Argentino de Informática y Salud, $37^{\circ}$ Jornadas Argentinas de Informática; 2008; Santa Fe (SF), Argentina; p. 1-12.

7. Haralick R; Shanmugam K; Dinstein I. Texture Features for Image Classification. Proceeding of IEEE Transaction on Systems, Man, and Cybernetis. 1973; 3(6): p. 610-621.

8. Quinlan JR. C4.5: Programs for Machine Learning. San Mateo (CA), USA: Editora Morgan Kaufmann; 1993.
9. Doria U. Introdução à Bioestatística: para simples mortais. São Paulo (SP), Brasil: Editora Elseiver; 1999.

10. Howarth P, Yavlinsky A, Heesch D, Rüger S. Medical Image Retrieval Using Texture, Locality and Colour. Lecture Notes from the Cross Language Evaluation Forum. 2005; p. 740749 .

11. Karkanis S, et al. Detecting Abnormalities in Colonoscopic Images by Textural Description and Neural Networks. Proceedings of Workshop on Machine Learning in Medical Applications, Advance Course in Artificial Intelligence; 1999; Chania, Greece; p. 5:59-62.

12. Ferrero CA, Lee HD, Cherman EA, Coy CSR, Góes JRN, Fagundes JJ, Wu FC. Utilização de Técnicas Computacionais para a Diferenciação Cólicos em Imagens Colonoscópicas. 56 Congresso Brasileiro de Coloproctologia: Rev Bras de Coloproct 2007; Curitiba (PR), Brasil; 27: p. 45-46.

${ }^{1}$ http://www.cs.waikato.ac.nz/ml/weka/.

${ }^{2}$ http://www.graphpad.com/.

Endereço para correspondência:

CARLOS ANDRES FERRERO

Laboratório de Bioinformática (LABI)

Universidade Estadual do Oeste do Paraná (UNIOESTE)

Parque Tecnológico Itaipu (PTI)

Av. Tancredo Neves, 6731

CEP: 85.856-970 / Caixa Postal: 39

Foz do Iguaçu - Paraná - Brasil

Fax: (45) 3522-3462

E-mail: anfer86@gmail.com 